\title{
Médiévales
}

Langues, Textes, Histoire

\section{La dynamique sino-japonaise (wakan) à l'époque de Heian}

The Sino-Japanese Dynamic (wakan) during the Heian Period

Ivo Smits

Traducteur : Alban Gautier

\section{OpenEdition}

\section{Journals}

Édition électronique

URL : https://journals.openedition.org/medievales/8043

DOI : 10.4000/medievales.8043

ISSN : $1777-5892$

\section{Éditeur}

Presses universitaires de Vincennes

\section{Édition imprimée}

Date de publication : 22 juin 2017

Pagination : $39-56$

ISBN : 978-2-84292-612-0

ISSN : 0751-2708

\section{Référence électronique}

Ivo Smits, « La dynamique sino-japonaise (wakan) à l'époque de Heian », Médiévales [En ligne], 72 printemps 2017, mis en ligne le 28 février 2019, consulté le 22 avril 2022. URL : http://

journals.openedition.org/medievales/8043; DOI : https://doi.org/10.4000/medievales.8043 
Ivo Smits

\section{La dynamique sino-japonaise (wakan) à l'époque de Heian}

La société de cour dans le Japon de Heian (794-1185) était, selon la formule consacrée, « un monde au-dessus des nuages », et l'attrait qu'exerce le Roman du Genji (Genji monogatari, ca 1008) ${ }^{1}$ - une œuvre littéraire qui évoque de manière emblématique les méditations, les succès et les angoisses de courtisans désœuvrés en quête de beauté - explique en partie pourquoi la littérature de Heian est principalement regardée comme l'expression d'une culture de cour hautement esthétisante, raffinée, émotive, introspective, et aux mœurs relâchées ${ }^{2}$. Cette réputation qui pèse sur les classiques de Heian n'est certes pas sans fondement, mais elle obscurcit la riche variété de la production textuelle de cette période.

L'étude qu'on va lire commencera par reconnaître que le contexte culturel du Roman du Genji n'était pas purement vernaculaire, mais au moins bilingue. Il convient en effet de regarder plus largement la culture littéraire de Heian comme une littérature faite de textes composés aussi bien en japonais (wabun) qu'en chinois ou en sino-japonais (que l'on désigne par l'expression kanbun) : le corpus entier de ces textes pourra ainsi être vu comme un tout organique. C'est pourquoi cet essai s'intéressera aussi aux dynamiques de la « japonité » et de la « sinité » dans la culture textuelle de la cour de Heian.

1. Pour une traduction française, voir MURASAKi ShiKIBU, Le Dit du Genji, trad. R. SIEFFERT, Paris, 1988, rééd. Lagrasse, 2011 [N.D.T.].

2. L'ouvrage qui a sans doute le mieux introduit cette idée en Occident est celui, classique, d'Ivan Morris : I. MORRIS, The World of the Shining Prince : Court Life in Ancient Japan, New York, 1964 ; trad. fr. M. CHARVET, La Vie de cour dans l'ancien Japon au temps du prince Genji, Paris, 1969. 


\section{La formation des canons}

Ce qui constitue aujourd'hui le canon de la littérature de Heian a pris forme à la fin du XIX siècle, et sa première apparition officielle remonte à la publication des premières histoires de la littérature de type moderne : l'Anthologie de la littérature nationale (Kokubungaku tokuhon, 1890) de Haga Yaichi (1867-1927), professeur à l'Université impériale de Tôkyô, ou encore la volumineuse Histoire de la littérature japonaise (Nihon bungakushi, 1890) de Mikami Sanji (1865-1939) et Takatsu Kuwasaburô (1864-1921). Ces publications étaient caractérisées par leur conception moderne des deux termes qui constituent le concept de «littérature japonaise ». L'idée même de « littérature » y était définie conformément à l'idée des « belles lettres », nouvellement importée depuis l'Occident. C'est ce dont témoigne la réinvention du mot bungaku, un terme initialement propre à l'historiographie chinoise classique, où il désignait l'étude des arts en général, qui en vint à désigner des textes qui, d'une manière ou d'une autre, donnaient accès à des univers intérieurs ou extérieurs par le biais de l'imagination: en d'autres termes, des textes relevant des genres épique, lyrique ou dramatique. Quant à la «japonité » de cette littérature, on la rapprochera des développements contemporains visant à faire de la langue japonaise une «langue nationale» (kokugo), capable de donner une cohérence à la nation japonaise que le régime Meiji (1868-1912) prétendait construire. La littérature japonaise devait donc être vue comme une «littérature nationale »(kokubungaku) : les nouvelles histoires de la littérature affirmaient ainsi que le Japon possédait une riche et longue tradition de genres véritablement littéraires; et elles en vinrent très vite à suggérer que les œuvres retenues dans le canon exprimaient des traits positifs du caractère national japonais ${ }^{3}$.

Lors de la mise en place de ces premiers canons littéraires modernes dans les années 1890 , la poésie et la prose en langue japonaise produites à partir de l'époque de Heian (794-1185) furent identifiées comme le premier corpus cohérent de textes relevant de l'étiquette «littérature japonaise ». Ainsi, parce qu'un grand nombre de textes composés dans la langue nationale, identifiés comme un socle fondamental d'une littérature nationale, avaient vu le jour précisément à cette époque, la culture de cour de Heian fut elle-même rétrospectivement identifiée comme un des principaux points d'origine de la culture nationale.

Le fait que cette même culture de cour a, pour sa part, semblé vouloir définir une certaine forme de «japonité »-en cela que les catégories de

3. Sur cette question, voir E. LOZERAND, Littérature et génie national : naissance d'une histoire littéraire dans le Japon du XIXe siècle, Paris, 2005. Je remercie Terada Sumie de m'avoir signalé cette référence en français. 
«Japon» et de «Chine»y étaient regardées comme distinctes - ne pouvait que renforcer cette assignation. De fait, bien des catégories qui en vinrent à être associées aux concepts de «Japon » et de «Chine » semblent avoir existé à la cour de Heian. Ainsi, les caractères chinois, qualifiés d' «écriture masculine » (otoko moji) et utilisés pour les textes publics, ou encore la peinture de style chinois (kara-e) apparaissant dans l'espace public, fonctionnaient comme l'inverse de l'écriture syllabaire japonaise (kana, et plus spécifiquement sa forme cursive ou hiragana), qualifiée d' « écriture féminine » (onnade) et réservée à des textes plus personnels, et donc « littéraires », illustrés de dessins « japonais » (yamato-e), voire de « dessins féminins » (onna-e) ; et ainsi de suite.

L'impact du canon constitué à la fin du XIX et au début du XX $\mathrm{X}^{\mathrm{e}}$ siècle est immense. Non seulement il a déterminé le choix des textes qui devaient être considérés par la suite comme des œuvres littéraires majeures, mais - ce qui est peut-être encore plus important - il a ancré dans les esprits l'idée que le japonais (classique ou moderne) était la seule langue dans laquelle une littérature avait pu exister. Cette marginalisation d'un immense corpus de textes sino-japonais fut en outre institutionnalisée par la création simultanée, à la fin du XIX ${ }^{\mathrm{e}}$ siècle, d'universités dans lesquelles les « études chinoises » (kangaku) constituaient un champ académique étudiant les textes composés en Chine, tandis que les «études de littérature nationale » étaient consacrées à l'étude des textes en japonais ${ }^{4}$.

Dans ce grand récit désormais traditionnel de l'histoire littéraire japonaise, l'époque de Heian est présentée comme un temps où la poésie en japonais (uta ou waka) a enfin accédé au statut d'art littéraire majeur, grâce à la première commande impériale d'une anthologie de waka, le Recueil de poèmes anciens et modernes (Kokin wakashû, aussi appelé Kokinshû, commandé en 905 et achevé vers 914$)^{5}$. Cette période est aussi vue comme celle où les récits de fiction et la littérature de mémoires arrivèrent à maturité, avec des chefs-d'œuvre tels que le Roman du Genji ou les Notes de chevet (Makura no sôshi, années 990) ${ }^{6}$. En bref, l'époque de Heian serait le moment où la poésie et la prose vernaculaires l'auraient emporté sur la domination de l'écriture sino-japonaise et où les modèles littéraires venus

4. M. KUROZUMI, «Kangaku: writing and institution authority », trad. D. LURIE, dans H. SHIRANE et T. SuZUKI éd., Inventing the Classics : Modernity, National Identity, and Japanese Literatures, Stanford, 2000, p. 201-219.

5. Pour une traduction française intégrale de la préface de cette anthologie, voir Le Monument poétique de Heian : le Kokinshû, trad. G. BonNEAU, vol. 1, Paris, 1933; on trouvera une sélection de quatre-vingts poèmes de cette même anthologie dans G. BONNEAU, Chefs-d'œuvre du Kokinshû, Paris, 1934 [N.D.T.].

6. Pour une traduction française, voir SEI SHÔNAGON, Notes de chevet, trad. A. BEAUjARD, Paris, 1966. Il s'agit de l'œuvre que les anglophones appellent The PillowBook [N.D.T.]. 
de Chine seraient devenus, soit des tremplins vers des genres littéraires indigènes, soit des repoussoirs auxquels il était nécessaire de résister. Beaucoup d'œuvres littéraires majeures en langue japonaise, mais aussi des formes littéraires vernaculaires identifiées comme «classiques » au Japon comme dans le reste du monde (le monogatari, ou littérature narrative, et le waka), sont bien des produits de la société de cour de Heian, et ce fait ne peut qu'influencer notre perception de la littérature de cette période. Or, s'il est vrai que la culture de cour était le principal espace de production de ces textes, l'assimilation pure et simple entre société de cour et canon littéraire tend à rétrécir notre vision de ce que la littérature pouvait alors recouvrir. En outre, une telle approche réduit l'essentiel de la littérature de Heian, qui comprend pourtant quatre siècles d'écriture, à quelques textes en langue vernaculaire et à quelques genres élaborés au sein de cercles proches de la cour pendant le siècle crucial qui va environ de 900 à l'an 1000. C'est en effet au début du $\mathrm{X}^{\mathrm{e}}$ siècle, après une période pendant laquelle la poésie et la prose sino-japonaise avaient joui de la faveur impériale, que le Kokin wakash û codifia la pratique et le développement formel de la poésie en japonais. Il servit ensuite de modèle à une série de vingt et une anthologies officielles jusqu'au XIV ${ }^{\mathrm{e}}$ siècle, toutes compilées selon le même principe de base. À côté des œuvres en prose comme les Contes d'Ise (Ise monogatari, $\mathrm{X}^{\mathrm{e}}$ siècle $)^{7}$ et le Roman du Genji, cette anthologie devint aussi une pierre de touche intertextuelle pour de nombreuses autres ouvres relevant de genres très divers.

Cependant, malgré la place éminente qu'occupent à juste titre des œuvres aussi célèbres, il convient de reconnaître que celles composées en prose n'occupaient qu'une place réduite au sein de la culture de cour du Japon de Heian. Les sources de l'époque contredisent donc le canon du XIX ${ }^{e}$ siècle. Elles révèlent en effet que la hiérarchie des genres plaçait alors au sommet les textes bouddhiques et confucéens (en chinois), les textes historiques chinois et les collections de poésie chinoise ; la poésie en japonais (waka) ne venait qu'ensuite; et tout en bas, on trouvait les œuvres narratives vernaculaires. Si le Roman du Genji fut « canonisé » dans les deux siècles qui suivirent sa composition et devint une véritable icône culturelle, on notera que l'importante bibliothèque de Fujiwara no Michinori (11061159) - homme d'État, savant et auteur de poèmes sino-japonais (kanshi) ne contenait pas un seul livre écrit en caractères vernaculaires japonais : tous étaient en chinois ou en sino-japonais (kanbun).

7. Pour une traduction française, voir Contes d'Ise, trad. G. RenondeAu, Paris, 1969 [N.D.T.]. 


\section{Systèmes d'écriture}

Le Japon de Heian était, pour dire le moins, une culture «biscriptuelle», ce qui implique qu'elle était aussi une culture bilingue ${ }^{8}$. Néanmoins, la juxtaposition de textes en écritures japonaise, ou kana, et sino-japonaise, ou kanbun, peut s'avérer trompeuse, car elle suggère une forme d'incompatibilité mutuelle entre les deux catégories. Or, il apparaît plutôt que ces oppositions binaires pouvaient se combiner et donner lieu à une synthèse (comme l'illustre par exemple le Wakan rôeishû, Recueil de poèmes à chanter en chinois et en japonais ${ }^{9}$ : voir fig. 1).

Le mot wakan est aussi utilisé pour dénoter toute une variété de rencontres entre des entités culturelles «japonaises » $(w a)$ et « chinoises » (kan). Ce terme, utilisé au moins depuis le $\mathrm{XI}^{\mathrm{e}}$ siècle, correspondait à l'origine à une énumération ( « le Japon et la Chine »), mais il est devenu par la suite un des fondements du discours critique et a servi à décrire la culture de cour de Heian comme un lieu d'opposition autant que d'interactions entre wa et kan. Or, les textes prenaient forme dans un environnement où l'on n'hésitait pas à mélanger les langues, les écritures, les sources et les modèles.

Le premier système d'écriture dont on disposa au Japon fut celui des idéogrammes chinois, un système où les éléments graphiques transmettent une signification (mana, littéralement «vrais noms », c'est-à-dire des logographes, appelés également kanji, autrement dit «l'écriture des Han ») (voir fig. 2).

Non seulement ces caractères furent utilisés pour écrire les textes sino-japonais (kanbun), mais on développa aussi des formes alternatives de notation pour les textes en japonais, formes réunies sous le terme man. yôgana (c'est-à-dire les kana tels que les utilise le Man.yôshî, texte du VIII ${ }^{\mathrm{e}}$ siècle) : les éléments graphiques y représentent des sons (kana, «noms provisoires ») plutôt que des significations. On les connaît aussi sous le nom de magana ou «kana authentiques » (c'est-à-dire inchangés dans leur forme) : ce sont des caractères qui représentent des sons (kana), mais qui ressemblent encore aux logographes (mana) dont ils descendent. L'étape suivante a vu le développement, au cours du $\mathrm{IX}^{\mathrm{e}}$ siècle, de deux séries de caractères syllabiques, toutes deux dérivées des man.yôgana. Le premier, connu sous le nom de katakana, déjà bien développé en 828 , était

8. Pour toute la section qui suit, voir C. SEELEY, A History of Writing in Japan, Leyde, 1991, p. 59-81.

9. Sur ce recueil, voir I. SMITS, « Song as Cultural History : Reading Wakan rôeishû », Monumenta Nipponica 55/2 (2000), p. 225-256, et 55/3 (2000), p. 399-427 ; ID., « The Way of the Literati : Chinese Learning and Literary Practice in Mid-Heian Japan », dans ADOLPHSON et al. éd., dans M. S. Adolphson, E. Kamens et S. Matsumoto éd., Heian Japan, Centers and Peripheries, Honolulu, 2007, p. 105-128. 


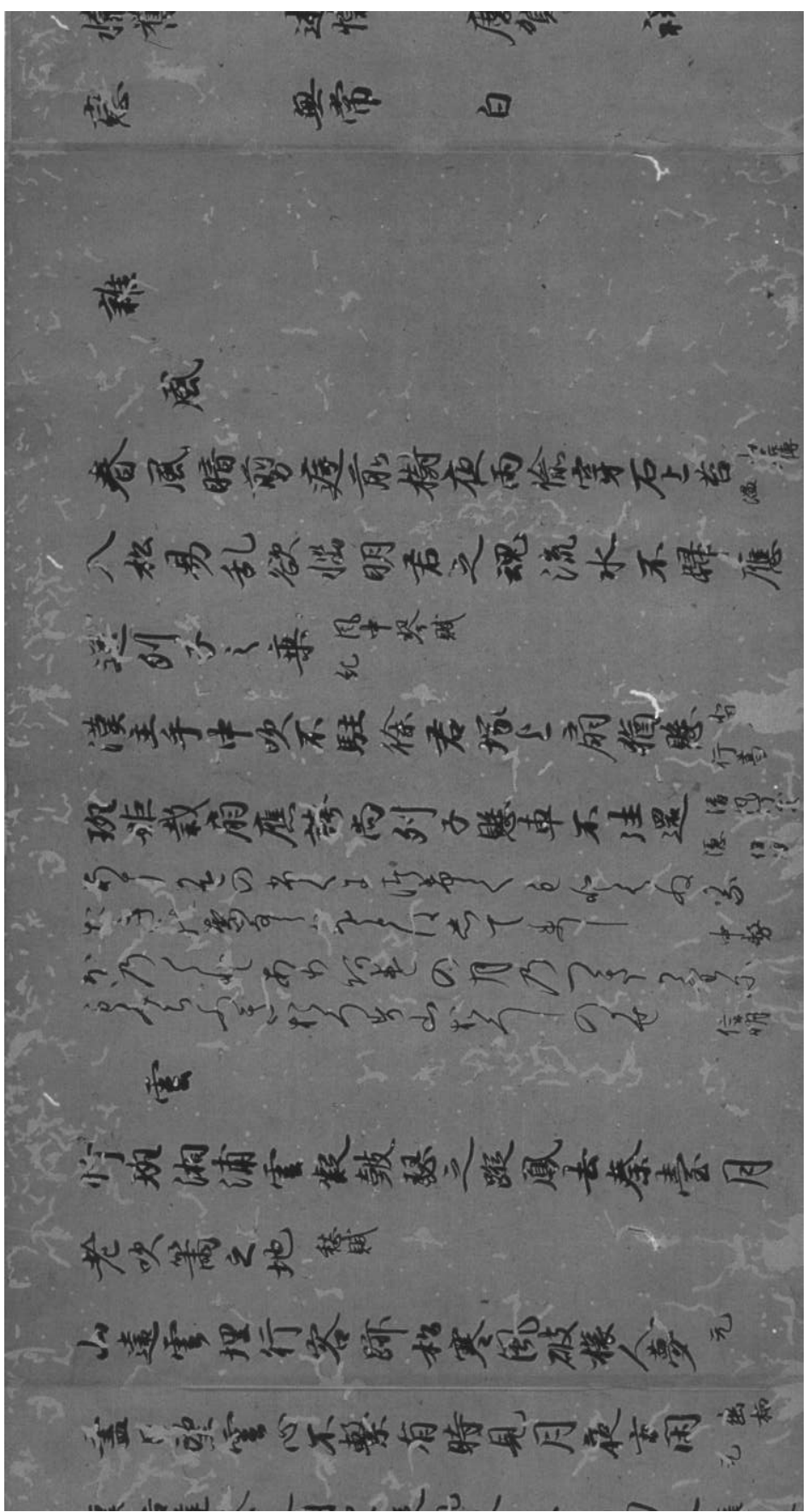




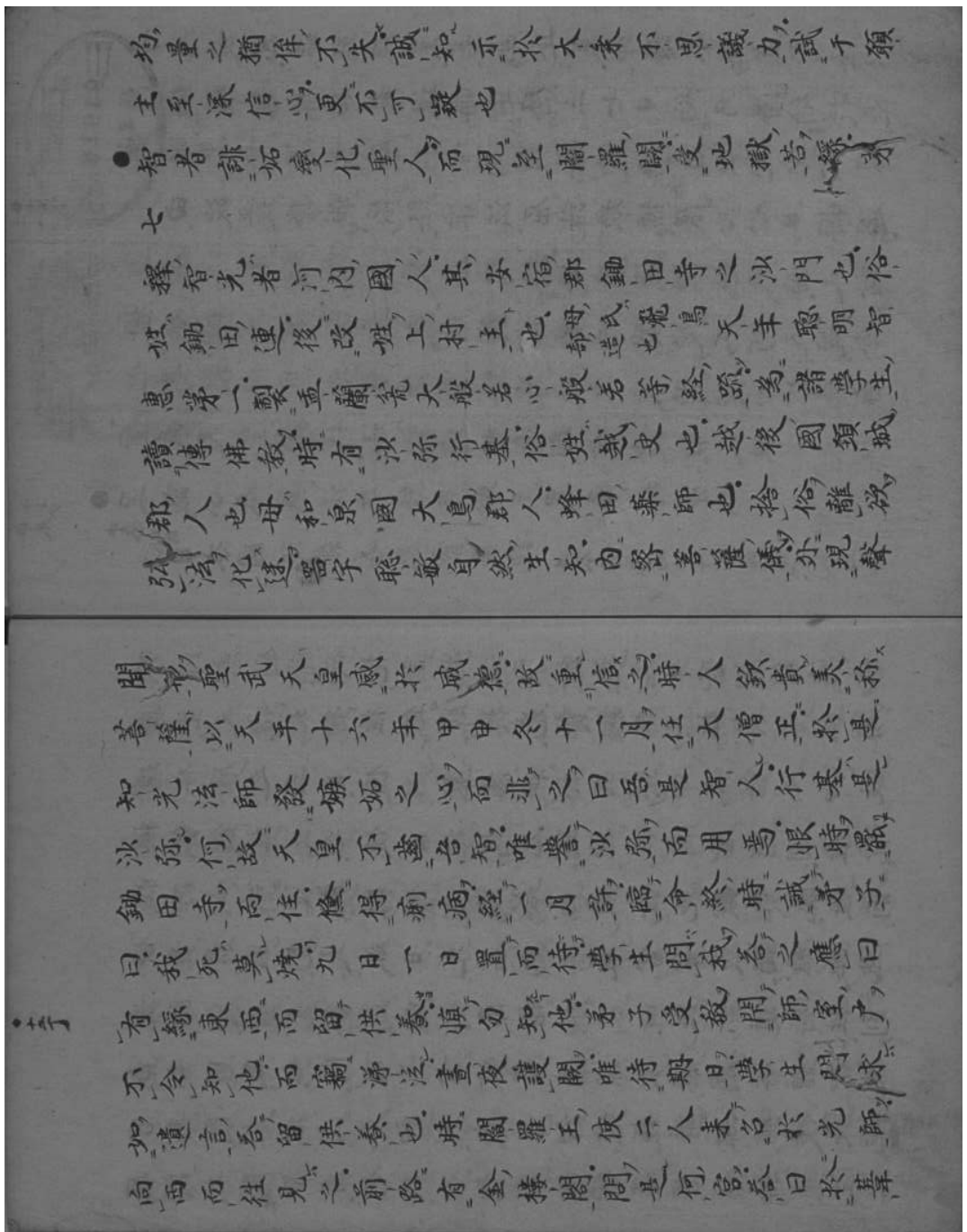

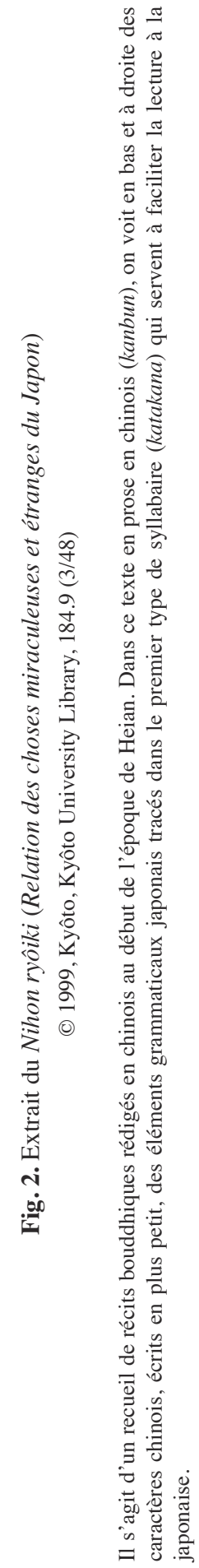


principalement utilisé dans les cercles monastiques et lettrés (bunshi), et servait surtout à la glose et à l'interprétation de textes chinois ; au XII ${ }^{\mathrm{e}}$ siècle au plus tard, on vit aussi des écrivains masculins composer des textes nouveaux en katakana. L'autre écriture syllabique, connue bien plus tard (au XVII ${ }^{\mathrm{e}}$ siècle) sous le nom de hiragana («kana lissés », c'est-à-dire cursifs), a acquis sa forme accomplie dans la seconde moitié du IX siècle (voir fig. 3).

Les éléments graphiques hiragana sont des formes extrêmement cursives de caractères chinois, utilisés pour représenter des sons. Chaque syllabe peut être représentée par des kana différents, exactement comme des caractères chinois variés avaient pu être utilisés pour noter une même syllabe dans les textes des $\mathrm{VII}^{\mathrm{e}}$ et $\mathrm{VIII}^{\mathrm{e}}$ siècles. Les textes en hiragana, dont le Kokin wakashû est le premier exemple important, tendent à la suppression complète des logographes. Or, c'est cette forme d'écriture qui allait symboliser le mieux la littérature de Heian : une écriture qui note des textes vernaculaires japonais et qui, visuellement autant que linguistiquement, évite toute référence à une « Chine ».

Pourtant, la réalité du champ textuel de Heian est bien plus complexe. Les scripteurs pouvaient (et ils ne s'en privaient pas) naviguer au sein d'un même texte entre différents systèmes d'écriture (logographes, kana), ainsi qu'entre les langues qu'ils notaient (chinois ou sino-japonais, japonais). En d'autres termes, les textes en langue vernaculaire et notés en écriture kana ne constituent qu'une des extrémités d'un spectre.

\section{Écritures et espaces genrés}

Vers la fin du $\mathrm{X}^{\mathrm{e}}$ siècle, les systèmes d'écriture semblent avoir assumé des identités genrées. Les hiragana furent explicitement désignés comme une «écriture de femme» ou «main de femme» (onnade). On continue certes à se demander si le terme onnade ne désignait pas un style calligraphique plutôt qu'un système d'écriture au sens strict, mais il reste qu'en pratique les hiragana et un rendu calligraphique «féminin » des caractères se combinent et laissent apparaître une conception selon laquelle l'écriture vernaculaire, singulièrement en prose, serait pour l'essentiel « féminine ». L'identification de l'écriture hiragana comme féminine a plus tard donné naissance à l'idée que le Japon de Heian était caractérisé par une stricte division entre d'un côté un monde masculin de l'orthodoxie et de la technique, tourné vers la tradition chinoise, et d'autre part un monde féminin de la créativité et de la fiction artistique en langue japonaise. Cette distinction a eu des répercussions importantes, car le domaine féminin s'est retrouvé associé au «Japon», et le domaine masculin à la «Chine ». L'idée de «Chine » subsumait les affaires d'État, les fonctions officielles, l'appropriation par le Japon des traditions classiques chinoises 


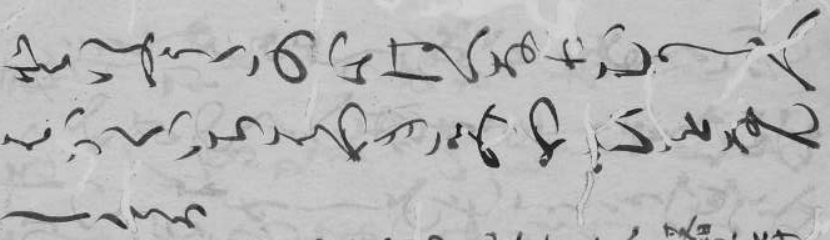

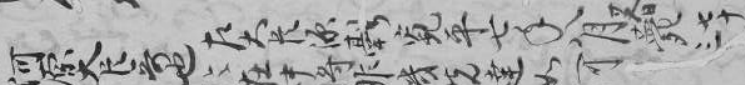
,

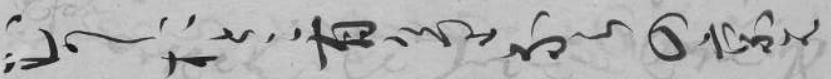

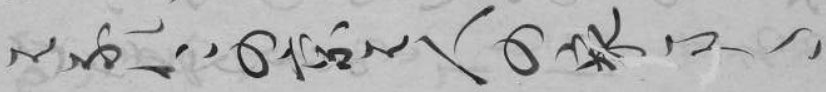

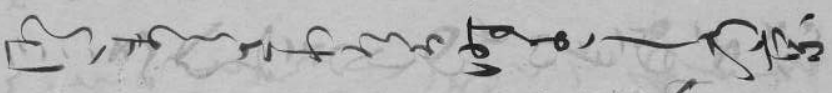

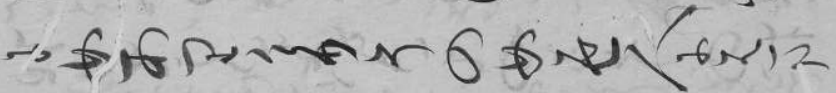

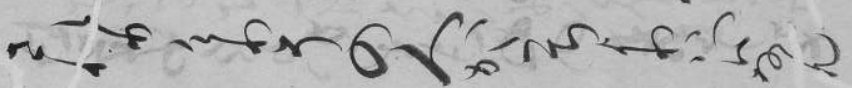

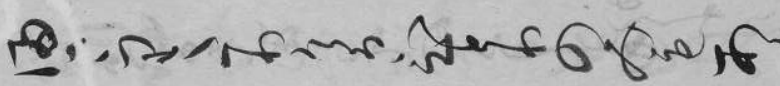

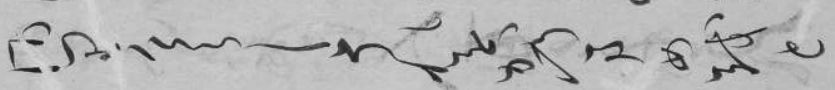

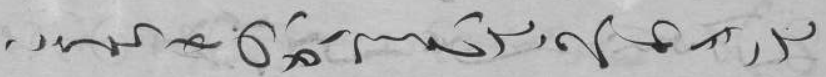

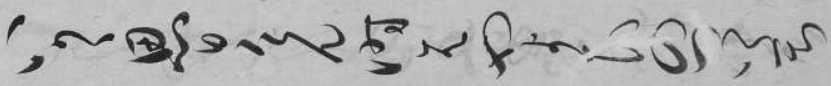
it 6 m

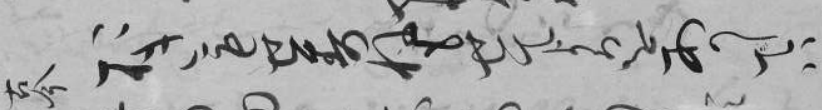
ho O Jum her fvis in

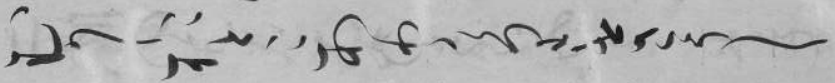

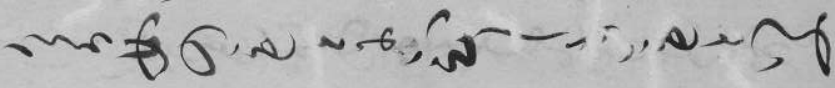
$\operatorname{arc} \theta+2+5$ 
de connaissance, de rationalité, etc. Au contraire, l'idée de «Japon » avait à voir avec la sphère personnelle (un adjectif qui permet d'éviter la notion anachronique de « privé »), les émotions, l'intuition, le lyrisme, la créativité artistique, etc. Soulignons cependant que la formulation explicite de ces identités genrées date d'une époque beaucoup plus tardive : elle n'apparaît en effet qu'au XVIII ${ }^{\mathrm{e}}$ siècle, lorsque le mouvement des « études nationales » (kokugaku) a vu dans la cour de Heian un foyer essentiel de l'identité culturelle. En d'autres termes, beaucoup de choses restent à élucider à propos des conceptions indéniablement genrées des systèmes d'écriture et des textes auxquels ils ont donné forme ${ }^{10}$.

Vers la fin du $\mathrm{X}^{\mathrm{e}}$ siècle, la cour de Heian ne se contentait pas de genrer l'écriture : elle genrait aussi les espaces physiques et sociaux. Un exemple bien connu est celui des peintures murales de la partie centrale de la résidence impériale (le Shishinden). Les historiens de l'art japonais qui travaillent sur l'époque de Heian distinguent entre « peinture chinoise » (kara-e) et «peinture japonaise» (yamato-e). Or cette distinction ne recouvre pas vraiment des différences de style, mais plutôt thématiques. Les « peintures chinoises » représentent des sujets réputés chinois : par exemple des sages chinois dans un bosquet de bambous, ou encore l'inquiétante « mer déchaînée » qui orne les portes coulissantes des appartements impériaux, avec ses étranges «créatures aux longs bras et aux longues jambes ${ }^{11}$ ». La «peinture japonaise » figure quant à elle des scènes qui se déroulent au Japon. Les secteurs du palais impérial ${ }^{12}$ utilisés pour des usages « officiels » et publics, et donc pour les affaires ritualisées, étaient généralement décorés de «peintures chinoises », tandis que les appartements de l'empereur (le Seiryôden) étaient habituellement agrémentés de «peintures japonaises ». Et même là, on peut repérer une distinction supplémentaire : l'appartement personnel de l'empereur (tennô) était orné de « peintures chinoises », tandis que, de l'autre côté de la même cloison, là où se tenaient les dames d'honneur de l'empereur, les parois étaient couvertes de «peintures japonaises ». Ces dernières pouvaient d'ailleurs à leur tour être subdivisées en «peintures féminines » (onna-e) et « peintures masculines » (otoko-e $)$, une distinction qui semble là aussi devoir être rapportée aux sujets représentés autant qu'au

10. Voir T. YodA, Gender and National Literature : Heian Texts in the Constructions of Japanese Modernity, Durham (NC), 2004, p. 81-110.

11. SEI SHÔNAGON, Notes de chevet..., p. 43 [N.D.T.].

12. Au centre du palais impérial était aménagée la résidence impériale proprement dite (appelée palais intérieur), entourée de murs. Cette résidence composée de pavillons était essentiellement destinée à la vie quotidienne de l'empereur. Voir à ce sujet l'article de M. MAURIN, «Le palais de Heian : sur les pas du Genji », Cipango, numéro hors-série, Autour du Genji monogatari, 2008 (https://cipango.revues.org/601). Je remercie Terada Sumie de m'avoir signalé cette référence en français. 
style - ce qui nous amène à établir un lien entre la «peinture féminine » et la fiction narrative ${ }^{13}$.

Pour résumer, à la cour de Heian, les notions de « chinois » et de « japonais » étaient largement identifiées à des notions de genre, qui à leur tour coïncidaient avec des fonctions plutôt qu'avec le sexe biologique. Les mots « homme » (otoko) et «femme» (onna) doivent par conséquent être compris comme signifiant plutôt « masculin » et «féminin ». Mais les hommes avaient la possibilité d'opérer selon des modalités « masculines » ou « féminines », en fonction des exigences particulières de chaque situation, ce que les femmes ne pouvaient normalement pas faire. Il faut en outre rappeler que cette répartition genrée des systèmes d'écriture, des espaces et des images, ainsi que le rôle éminent qu'ont joué les femmes dans la création et le développement de nouveaux genres littéraires vernaculaires, n'a véritablement commencé qu'à la fin du $X^{\mathrm{e}}$ siècle.

Cette répartition genrée est liée à l'émergence, au cours du $\mathrm{X}^{\mathrm{e}}$ siècle, d'un nouvel idéal aristocratique, qui a pu être décrit à juste titre comme anti-professionnel, anti-méritocratique et, dans une certaine mesure, antiintellectuel ${ }^{14}$. L'ascension de la Maison des Régents (sekkanke) issus du lignage ou « clan » (uji) Fujiwara a principalement reposé sur un système où le pouvoir était négocié et obtenu à travers des mariages : pour cette raison, le centre de gravité politique s'est déplacé vers les secteurs supposément privés, ou «arrières », du complexe palatial (kôkŷu), où précisément le système d'écriture dominant était les kana, et où la culture privilégiait les productions en langue vernaculaire. Ces textes en kana mettaient de fait l'accent sur une écriture introspective, exprimant l'état intérieur de leurs auteurs et protagonistes, qu'il s'agisse de poésie, de prose, ou d'une combinaison inextricable des deux. Le rôle central joué par les auteurs féminins dans le développement de la fiction narrative et de l'écriture de soi en langue japonaise - phénomène sans équivalent dans d'autres cultures littéraires - est sans aucun doute lié aux opportunités nouvelles que créait cette situation politico-culturelle à la cour.

Les interprétations ultérieures de cette répartition genrée des systèmes d'écriture ont donné naissance à l'idée erronée d'un « tabou » de l'écriture chinoise chez les femmes de l'époque de Heian. Il existe en réalité des indices qui vont dans le sens inverse, même s'il est vrai que l'éducation formelle en chinois est restée une prérogative masculine tout au long de la période. L'étude plus précise de l'arrière-plan familial de plusieurs femmes,

13. K. ChINo, "Gender in Japanese Art», dans J. S. Mostow, N. BRYSON et M. GRAYBiLl éd., Gender and Power in the Japanese Visual Field, Honolulu, 2003, p. 17-34.

14. J. S. Mostow, "Mother Tongue and Father Script: The Relationship of Sei Shônagon and Murasaki Shikibu to Their Fathers and Chinese Letters », dans R. L. COPELAND et E. RAMIREZ-CHRISTENSEN éd., The Father-Daughter Plot : Japanese Literary Women and the Law of the Father, Honolulu, 2001, p. 115-142 (p. 135). 
aujourd'hui associées au développement de la fiction narrative en kana et à la production de littérature de cour, montre qu'elles appartenaient à des familles de lettrés qui exerçaient souvent les fonctions de gouverneurs de province (zuryô). Murasaki Shikibu (973 ?-1014 ?) elle-même, ainsi que Akazome Emon (960 ?-1045 ?), Sei Shônagon (966 ?-après 1017), et la fille de Sugawara no Takasue (Sugawara no Takasue no Musume, 1008- ?), avaient toutes dans leur famille une tradition de savoir chinois, et à ce titre elles possédaient toutes à des degrés variés une certaine maîtrise de l'écriture chinoise.

\section{Références chinoises}

Le Japon de Heian faisait partie de l'Asie orientale : l'un des faits qui le montre le mieux est l'omniprésence de certains textes clés de la tradition littéraire chinoise, qui ont servi de référence tout au long de la période. Il serait trop long d'en dresser le catalogue complet, mais deux œuvres peuvent être mentionnées parmi d'autres: l'Anthologie des belles lettres (chinois Wenxuan, japonais Monzen, début du VI siècle) ${ }^{15}$ et l'Anthologie des poèmes de Bai Juyi (chinois Baishi wenji, japonais Hakushi monjû ou Hakushi bunsû, 839). La grande importance de ces deux ouvrages apparaît bien dans un passage des Notes de chevet (années 990), où la dame de compagnie Sei Shônagon écrit : « les textes en chinois sont : l'Anthologie des poèmes de Bai Juyi, l'Anthologie des belles lettres, [...] des dédicaces religieuses, des suppliques adressées au trône, des requêtes officielles rédigées par de savants professeurs ». Les deux ouvrages sont présents dans le plus ancien catalogue conservé des collections de livres continentaux de la bibliothèque impériale, le Catalogue des livres existant au Japon (Nihonkoku genzaisho mokuroku, réalisé peu après 891).

L'Anthologie des belles lettres, compilée sous le patronage du prince Xiao Tong (501-531), héritier désigné de la dynastie Liang, est classée par genres littéraires, et il est probable que ce trait ait contribué à en faire l'ouvrage canonique qu'elle est devenue à travers toute l'Asie orientale, et un manuel de base à l'Office des Études Supérieures (Daigakuryô) au Japon. Il s'agit à la fois d'un gigantesque recueil couvrant presque un millénaire, avec 130 auteurs et 761 textes en vers ou en prose, et d'un système de classification qui distingue 37 catégories génériques. Cette structure a permis à l'anthologie d'imposer des notions de genres littéraires dans la pensée littéraire chinoise, et elle devint une lecture obligatoire pour

15. On trouvera une sélection de textes traduits en français dans G. MARGOULIÈs, Le «Fou» dans le Wen Siuan : étude et textes, Paris, 1926. Il existe une traduction anglaise intégrale: Wen Xuan, or Selections of Refined Literature, trad. R. KNECHTGES, 3 vol., Princeton, 1982-1996 [N.D.T.]. 
toute personne formée aux classiques chinois - c'est-à-dire pour quiconque souhaitait obtenir un poste dans la bureaucratie d'État. Au Japon également, l'Anthologie des belles lettres fut fortement étudiée, ainsi que les nombreux commentaires apparus dès les années qui suivirent sa compilation. Avec ses trente volumes, elle était suffisamment exhaustive pour qu'on puisse croire qu'elle représentait la totalité de la tradition de ce qu'on appellerait plus tard la « littérature des Six Dynasties ${ }^{16} »$ : le fait que Xiao Tong avait réalisé une « sélection » semble avoir échappé à la plupart des lecteurs de l'époque de Heian.

Si le florilège que constituait l'Anthologie des belles lettres était prestigieux, on peut dire que les œuvres du poète chinois Bai (ou Bo) Juyi (772-846) étaient réellement populaires. Il n'y a pas de succès littéraire international dans l'Asie orientale classique que l'on puisse comparer à la manière dont Bai Juyi conquit ses lecteurs japonais. En 838, le gouverneur local de Dazaifu - le principal port japonais pour les contacts avec le continent asiatique - découvrit dans une cargaison venue de Chine un livre contenant des textes de Bai Juyi en vers et en prose. Il fit remettre l'ouvrage à l'empereur et cette démonstration d'expertise littéraire lui valut, comme il se devait, une promotion hiérarchique. Bai Juyi était arrivé au Japon et il devait y rester. Cette découverte fit naître un véritable emballement poétique parmi les lettrés japonais. Très vite, on se mit en quête d'une édition complète des œuvres de Bai. Le moine Egaku (actif entre 835 et 864), qui assurait pratiquement à lui seul une sorte de service de navette (on lui connaît quatre voyages sur le continent), joua un rôle important en tant que fournisseur. Lors de son deuxième voyage en Chine, il passa deux mois de l'année 844 à copier un recueil des œuvres de Bai au temple Nanchan de Suzhou. C'était cinq ans plus tôt que Bai lui-même avait remis sa propre version de ses œuvres à l'entrepôt de sutras du même temple : ce dépôt constituait alors l'une des trois seules copies des œuvres complètes de Bai disponibles en Chine. C'est cette version autorisée qui fut copiée et rapportée au Japon par Egaku. À peu près au même moment, un autre moine japonais, Ennin (794-864), qui résidait alors à Chang'an, la capitale de la Chine à l'époque des Tang (618-906), acheta lui aussi un «Recueil de la poésie de Bai en six volumes ». Après que les Japonais eurent officiellement décidé, en 894, de ne plus envoyer d'ambassades officielles en Chine, la cour chinoise prit l'habitude d'interroger les moines japonais qui voyageaient dans leur empire afin de se tenir au courant des événements récents au Japon. Lorsque ces moines étaient reçus en audience par l'empereur de Chine, le protocole incluait toujours une question sur les livres chinois connus au Japon, et les moines ne manquaient jamais de mentionner les œuvres de Bai Juyi. Bai

16. La période des « Six Dynasties » va traditionnellement de la chute de la dynastie Han en 220 de notre ère à la réunification de la Chine par la dynastie Sui en 589 [N.D.T.]. 
lui-même fut averti de son succès : dans un post-scriptum à un exemplaire de ses œuvres complètes, il mentionna que des copies étaient disponibles en Corée et au Japon. On sait en outre par des textes que, dans l'archipel, des lettrés japonais décoraient leur maison avec le portrait de Bai.

Les femmes aussi appréciaient la lecture et la récitation des poèmes de Bai. Dans ses Notes de chevet, Sei Shônagon insère à l'occasion quelques vers. Un passage bien connu est celui où l'impératrice Teishi (ou Sadako, 977-1000) fait référence à « la neige sur pic Xianglu », ce à quoi Sei répond en faisant relever les stores, laissant apparaître la neige dans le jardin : ce geste montre sa familiarité avec le distique de Bai, « Je lève le store pour contempler / la neige sur le pic Xianglu ${ }^{17} »$. On peut certes soutenir que des références à des vers aussi célèbres de Bai Juyi ne constituent pas nécessairement la preuve que les femmes lisaient réellement ses poèmes; mais il semble bien qu'une certaine fréquentation de son œuvre était considérée comme un atout. Ainsi, l'impératrice Shôshi (ou Akiko, 988-1074) est connue pour avoir activement étudié sa poésie sous la tutelle de sa dame de compagnie Murasaki Shikibu, l'auteure du Roman du $\mathrm{Genji}^{18}$. On notera ici que les séances d'étude de l'impératrice n'étaient pas « secrètes », comme certaines traductions pourraient le laisser penser, mais plutôt « informelles ».

La façon dont la poésie de Bai éclipsa celle de ses contemporains d'époque Tang dans la formation de l'univers poétique de Heian est tout à fait remarquable : elle ne peut pas être considérée comme un simple reflet du canon chinois. Il reste que la raison la plus probable du succès de Bai Juyi au Japon est son immense popularité en Chine même. Les moines de Heian qui voyageaient sur le continent ne pouvaient manquer de voir que tous les Chinois semblaient lire Bai Juyi. Enfin, la langue claire de Bai et la facilité relative avec laquelle on pouvait lire ses poèmes ont sans aucun doute contribué à son immense succès.

Les lecteurs de Heian aimaient Bai Juyi, mais ils n'ont pas nécessairement saisi toutes les dimensions de son œuvre. L'intérêt de Heian pour les «nouvelles ballades » (chinois xinyuefu, japonais shingafu) de Bai en est un exemple fascinant. Ce genre promu par Bai Juyi consistait en d'assez longs poèmes, de forme relativement libre, qui critiquaient en termes simples les injustices sociales et politiques. Bai avait classé ses «nouvelles ballades » à côté de ses « poèmes de remontrance et d'instruction » (chinois fengyushi, japonais fûyushi), et considérait que leur efficacité en vue d'un redressement moral était une de ses contributions les plus importantes à la poésie. Le goût de Heian pour des ballades aux thèmes souvent explicitement

17. SeI Shônagon, Notes de Chevet..., p. 293 [N.D.T.].

18. MuRASAKi ShIKIBU, Journal, trad. R. SiefFerT, Paris, 1978, p. 15-16 (rééd. Lagrasse, 2011) [N.D.T.]. 
politiques est assez étrange, car s'il est vrai que la noblesse japonaise était férue de culture chinoise, ses opinions politiques étaient diamétralement opposées à celles qu'expriment les ballades. Les courtisans de Heian ne montraient guère de compassion pour les classes les plus humbles, ils ne voyaient pas pourquoi l'administration aurait dû être (comme on l'affirmait en Chine) une question de compétence plutôt que de naissance, et ils ne pensaient pas que le devoir du détenteur d'un poste gouvernemental était de signaler à l'attention du monarque les éléments dysfonctionnels à l'intérieur du royaume. En fait, même si les courtisans de Heian voyaient bien que Bai lui-même avait classé ses «nouvelles ballades » dans le genre de la poésie politique, il est clair qu'en règle générale ils ne se souciaient guère du message de ces poèmes: ils s'intéressaient surtout à leurs passages descriptifs, qu'ils imitaient dans leurs propres compositions. Et pourtant les ballades étaient réellement populaires. Ainsi, quand Murasaki Shikibu étudiait avec son impératrice, les ballades de Bai étaient un des matériaux de lecture ${ }^{19}$. Et lorsque l'on sélectionnait des extraits de poèmes de Bai pour les copier, par exemple pour les offrir en cadeau, on choisissait presque toujours les «nouvelles ballades ». Les Japonais organisèrent même des sessions d'étude pour discuter ces poèmes. Aussi, lorsque des nobles de Heian parlaient de «ballades », ils se référaient toujours aux «nouvelles ballades » de Bai Juyi. Les lecteurs de poésie chinoise de cette période préféraient les textes simples. La langue claire des «nouvelles ballades » explique donc en grande partie leur popularité dans le Japon de Heian.

Bai Juyi et sa poésie sont très présents dans le Roman du Genji. Son Chant des regrets éternels (chinois Changhenge, japonais Chôgonka ${ }^{20}$ ) est explicitement mentionné par Murasaki Shikibu comme un modèle pour la relation entre la mère du Genji et l'empereur. Lorsque plus tard le Genji décide de s'imposer un exil à Suma, il emporte avec lui un exemplaire du «recueil de poèmes » (sous-entendu, ceux de Bai Juyi) et en récite fréquemment des extraits; et à l'instar du poète chinois lors de son propre exil, le Genji emporte également une cithare (kin), un instrument typiquement « chinois $»^{21}$. Ce détail nous permet de signaler

19. Pourtant l'historienne Maruyama Yumiko a récemment affirmé que le choix des nouvelles ballades de Bai Juyi était un choix consciemment politique de la part de Murasaki Shikibu, tutrice de la jeune impératrice. Selon elle, la maison Fujiwara, dont provenait l'impératrice, estimait que le potentiel politique de Shôshi en tant que « mère-impératrice » (bokô, c'est-à-dire mère d'un futur empereur apparenté aux Fujiwara) exigeait un programme éducatif approprié. Les ballades de Bai auraient ainsi été un élément de l'éducation proprement politique de Shôshi. Voir Y. MARUYAMA, Sei Shônagon to Murasaki Shikibu : Wa-Kan konkô no jidai no miya no nyôbô, Tôkyô, 2015, p. 94.

20. Pour une traduction française, voir BAI JUYI, Chant des regrets éternels et autres poèmes, trad. G. JAEGER, Paris, 1992 [N.D.T.].

21. Les références à « la Chine » dans le Roman du Genji sont étudiées par D. PoLLACK, The Fracture of Meaning : Japan's Synthesis of China from the Eighth through the Eighteenth 
que la dialectique Chine-Japon (wakan) était aussi véhiculée par des objets comparables à l'instrument de musique emporté en exil par le Genji. Ses relations avec les femmes peuvent aussi être exprimées à travers des objets : par exemple, la Dame Akashi (Akashi no kimi) cherche à séduire le Genji à l'aide d'objets de style chinois (un coussin de brocart, une cithare, de l'encens) qui la représentent lorsque, en son absence, il pénètre dans ses appartements $^{22}$.

Le Roman du Genji est souvent présenté comme le produit d'une culture de cour éminemment japonaise. Pourtant, cette culture de cour a toujours opéré dans le cadre d'une interaction dynamique entre des éléments culturels associés à «la Chine». De fait, cette «Chine» bien souvent anhistorique que les courtisans japonais avaient intégrée à leur propre univers était autant leur propre création - ce pourquoi on parle parfois d'une «Chine-dans-le-Japon »- qu'une entité politique et culturelle bien réelle située sur le continent. Cette Chine n'était pas vue comme opposée au Japon, mais plutôt comme une partie intégrante de celui-ci. Si l'on peut effectivement parler d'une dialectique wakan au sein de la culture de cour de Heian, il nous faut reconnaître qu'il ne s'agit pas tant de la « synthèse des contraires » que d'un processus dynamique de fusion d'éléments différents mais pas nécessairement antithétiques. De plus en plus de chercheurs s'ouvrent aujourd'hui à la riche présence, dans le Roman du Genji, de gestes se référant à cette « Chine », ainsi qu'à leur incorporation au contexte de la vie de cour japonaise. Il nous faut donc affirmer que le monde qu'habitait le Genji était, à l'instar du monde qui l'avait créé, tout à fait unique mais certainement pas étranger au sein de l'Asie orientale ${ }^{23}$.

Texte traduit de l'anglais par Alban Gautier

Centuries, Princeton, 1986, p. 55-76.

22. Dans le livre sur la visite du Genji au «Hatsune » : voir sur ce point KaWAzoE F., Karamono no bunkashi : hakuraihin kara mita Nihon, Tôkyô, 2014, p. 84-88 (MURASAKI SHIKIBU, Le Dit du Genji..., t. 1, livre 23, « Prime chant », p. 478 [N.D.T.]).

23. Nous recommandons également aux chercheurs non japonophones les ouvrages suivants : R. BORGEN, « The Politics of Classical Chinese in the Early Japanese Court », dans D. R. KNeChtges, E. VAnCE éd., Rhetoric and the Discourses of Power in Court Culture : China, Europe, and Japan, Seattle, 2005; W. DENECKE, Classical World Literatures: Sino-Japanese and Greco-Roman Comparisons, Oxford, 2014 ; E. KAMENS. «Terrains of Text in Mid-Heian Court Culture », dans M. S. Adolphson, E. KAMENS, S. Matsumoto éd., Heian Japan, Centers and Peripheries, Honolulu, 2007, p. 129-152 ; T. LAMARre. Uncovering Heian Japan : An Archeology of Sensation and Inscription, Durham (NC), 2000 ; H. C. MCCullough, Brocade by Night : «Kokin Wakash $\bar{u} »$ and the Court Style in Japanese Classical Poetry, Stanford, 1985 ; A. SAKAKI, Obsessions with the Sino-Japanese Polarity in Japanese Literature, Honolulu, 2006 ; M. URY, « Chinese Learning and Intellectual Life », 
Ivo Smits - Université de Leyde

\section{La dynamique sino-japonaise (wakan) à l'époque de Heian}

Cet essai explore la dynamique de la " japonité " (wa) et de la " sinité " (kan) au sein de la culture textuelle de la cour japonaise de Heian (794-1185). II considère que cette culture littéraire est constituée de textes aussi bien japonais que chinois, et envisage l'ensemble de ce corpus littéraire comme un tout organique. Le contexte culturel de cette œuvre classique de la littérature japonaise qu'est le Roman du Genji apparaît donc comme bilingue.

Bai Juyi - Genji - Heian - Japon - japonité - littérature sino-japonaise - Sei Shônagon - sinité

\section{The Sino-Japanese Dynamic (wakan) during the Heian Period}

This essay explores the dynamics of "Japaneseness" (wa) and "Chineseness" (kan) in the textual culture of the Japanese Heian court (794-1185). It regards this literary culture as a literature of texts in both Japanese and in Chinese, and sees the overall corpus of these texts as an organic whole. As such, the cultural context for the Japanese classic The Tale of Genji is understood to be a bilingual one.

Bai Juyi - Chineseness - Genji - Heian - Japan - Japaneseness - Sei Shônagon - Sino-Japanese Literature 
